\title{
A new formula for BCG scar and tuberculin test response
}

\begin{abstract}
Back ground: Tuberculosis is an important disorder in the world especially in developing and undeveloped regions, and the Bacille Calmette-Guérin (BCG) vaccination plays an important role in controlling this health problem. The rates of positive Purified protein derivative (PPD) test reactions and BCG scarring after BCG vaccination vary between surveys and different regions in the world.
\end{abstract}

Objective: This study was done to evaluate BCG scars and PPD response after BCG vaccination in Birjand, Iran.

Materials and methods: In this cross sectional descriptive analytical study, 120 children were enrolled. First, we measured BCG scars diameters and then 0.1 milliliter of standard 10 unit Purified protein derivative (PPD) solution was injected intradermally. After 72 hours, biggest indurations diameters were measured. Data were analyzed by using SPSS package, Chi Square and logistic regression analysis tests.

Results: 54 cases out of a total 120 cases were male and 66 cases were female. 38 cases $(32 \%)$ had no BCG scar and PPD test was negative in these cases. PPD test indurations diameters were lesser than 3 millimeters in 57 cases (48\%) and BCG scars diameters were lesser than 2 millimeters in 67 cases $(56 \%)$. The mean of BCG scars diameters were 1.95 millimeters in male cases and 1.46 millimeters in female cases. Mean of PPD SKIN test indurations diameters were 4.5 millimeters in male cases and 3.6 millimeters in female cases.

Conclusion: Consistent with these results, there is a significant positive relationship between tuberculin test indurations and BCG scar diameter. According to this formula (PPD test indurations diameter $=1.5+1.5 \times \mathrm{BCG}$ scar diameter), we can predict PPD test indurations in accordance with BCG scar diameter in 4 year children.
Volume 5 Issue 5 - 2017

\section{Reza Ghaderi}

Department of Dermatology, Birjand University of Medical Sciences, Iran

Correspondence: Reza Ghaderi, Department of Dermatology, Faculty of Medicine, Birjand University of Medical Sciences, Moallem St, Birjand, Iran, Medical Toxicology Research Center, Mashhad University of Medical Sciences, Mashhad, Iran, Tel 985632000000,Email rezaghaderi@yahoo.com

Received: January 01, 1970 | Published: June 22, 2017

Keywords: BCG scars, PPD, tuberculosis, vaccination

\section{Introduction}

Tuberculosis is an important disease in the world especially in developing and undeveloped regions and PPD test is the standard test for diagnosis of this infectious disease..$^{1-3} \mathrm{BCG}$ vaccination has proven to be the most effective technique for controlling tuberculosis in the world especially in developing countries. ${ }^{1}$ The BCG vaccination is the most extensively used immunization worldwide, nevertheless it can effect in the correct interpretation of the PPD test. ${ }^{3}$ The rates of positive Tuberculin Test responses and BCG scar after BCG vaccination vary between surveys and different regions in the world. ${ }^{2-13} \mathrm{PPD}$ test after vaccination can evaluate the immunogenic activity and protective efficacy of the, BCG immunization at birth is common in China. In a multi-site prospective cohort study, the immunological reactivity against BCG is evaluated in four prefectural cities in China. ${ }^{1}$ According to their results, not only the BCG vaccination among newborn infants in China causes satisfactory immune response, but also BCG scars provide a useful marker of immunization response in Chinese infants. BCG scarring and PPD reactivity possibly linked to better child survival in low-income countries. Therefore, in a study, determinants for Tuberculin test reaction and scarring was investigated in GuineaBissau. $^{2}$ In the multivariable analyses of BCG-vaccinated children evaluated at 6 months of age, monitoring of vaccination technique and type of BCG vaccine were important. It is concluded that vaccination technique and BCG strain are important for Tuberculin test reaction and scarring in response to BCG vaccination. Considering that these responses are associated with better infant survival, the importance of monitoring vaccination technique and of different $\mathrm{BCG}$ strains must be assessed with regard to infant mortality. PPD reactivity and BCG scarring have been argued as valid markers of BCG effectiveness for prevention of tuberculosis. ${ }^{2,8-16}$ We therefore aim to evaluate $\mathrm{BCG}$ scars and Tuberculin test response after BCG vaccination in 4 year children in Birjand City, Iran (exactly 4 years old).

\section{Materials and methods}

In this cross sectional study, 120 children were recruited. 66 (55\%) out of 120 children were female while 54 (45\%) children were male. Mean age of children was 4 years. Written informed consent was obtained from all participating families. First, we measured BCG scars diameters with a ruler in children and then, we used a Tuberculin test with purified protein derivative (PPD) at 4 years age. PPD $(0.1 \mathrm{ml}$, institute of Pasteur, 0561) was used intradermaly in the ventral aspect of the left forearm. The readings of the Tuberculin Test reaction were done by measuring diameters with a ruler after $48-72 \mathrm{~h}$. Data were analyzed using SPSS package.

\section{Results}

54 cases out of a total of 120 cases were male while 66 cases were female. 38 cases (32\%) had no BCG scar and PPD test was negative in this cases. Tuberculin Test indurations diameter were lesser than 3 millimeters in 57 cases $(48 \%)$ and BCG scars diameter were lesser than 2 millimeters in 67 cases $(56 \%)$. The mean of BCG scars diameter were 1.95 millimeters in male cases and 1.46 millimeters in female cases. 
Mean of Tuberculin Test indurations diameter were 4.5 millimeters in male cases and 3.6 millimeters in female cases. Consistent with these results, there is a significant positive relationship between tuberculin test indurations and BCG scar diameter. According to this formula (PPD test indurations diameter $=1.5+1.5 \times \mathrm{BCG}$ scar diameter $)$, we can predict PPD test indurations in accordance with BCG scar diameter in 4 year children.

\section{Discussion}

BCG vaccination is the most effective technique to control tuberculosis in childhood. ${ }^{1}$ To prevent tuberculosis, BCG vaccination is prescribed at birth in Iran and many other countries. ${ }^{2,7-10}$ PPD testing after immunization is often used to assess the protective efficiency of the BCG. ${ }^{1,2,4}$ The rates of positive PPD responses and BCG scarring after BCG vaccination vary between surveys and different regions in the world ${ }^{2,5-14}$ and a BCG vaccination $\mathrm{n}$ does not constantly result in a BCG scarring. ${ }^{2,15}$ On the other hand, there has not been a global concordance between the PPD test and the QuantiFERON-TB-Gold test in the diagnosis of tuberculosis. ${ }^{12,13}$ Recently a study showed that general concordance between PPD test and QuantiFERON-TBGold test in the diagnosis of latent tuberculosis in a group of health care workers was poor. The use of each of these tests is related to the outbreak of tuberculosis and the BCG vaccination basis. ${ }^{12}$ The extent of the PPD test and BCG scarring changes with the content of live bacilli of BCG. ${ }^{2}$ Another study investigated about relationship between diameter of BCG scars and effect of PPD response and also determined whether vitamin A combined vitamin D supplementation influences the immune reaction to BCG revaccinated in Chinese infants. ${ }^{3}$ As a result, there was a prominent correlation between the BCG scars diameter and the tuberculin test response, which shows BCG scar formation, may come useful in assessment of the effect of tuberculosis prevention. Vitamin A combined Vitamin D supplementation may modulate immune reaction in BCG revaccination. This may make a positive contribution to the prevention of childhood tuberculosis. ${ }^{3}$ The PPD test is extensively used to diagnosis Mycobacterium tuberculosis and evaluates latent tuberculosis cases, but the definition and the significance of a positive test in very young children is not still clear. ${ }^{4}$

In a study investigated the role of $\mathrm{BCG}$ vaccination and/or exposure to environmental Mycobacteria in PPD test reactivity. To achieve this goal, the PPD test was compared in children who either received BCG vaccination at birth or were BCG naïve at $4(1 / 2)$ months of age. Nearly half of the BCG vaccinated children had a positive PPD test ( $>$ or $=5 \mathrm{~mm}$ ) whereas all the $\mathrm{BCG}$ naïve children were non-reactive, proving that recent $\mathrm{BCG}$ vaccination influences PPD test reactivity. Naïve children were then vaccinated at $4(1 / 2)$ months of age and a PPD test was repeated at 20-28 months of age. The results suggested that the timing of BCG vaccination had little effect by this age. "Mycobacterial specific IFNgamma responses were greater in PPD test responders than in non-responders". The size of indurations did not associate with IFNgamma, but the IFNgamma: IL-10 ratio positively correlated with PPD test indurations declaring that the relationship between PPD induced IFNgamma and IL-10 in the peripheral blood may be an important tool in controlling PPD test reactivity. Collectively these data make more information available about the interpretation of a positive response and the regulation of PPD test in early life. ${ }^{4}$ PPD test is an easily performed, reproducible and low cost test. ${ }^{6}$ A cross-sectional epidemiological study was done to distinguish the PPD reactivity in both BCG vaccinated and unvaccinated population and to find the variables (BCG vaccination, the number of BCG scars, age, sex) influencing PPD reactivity. Its purpose was also to find out the annual risk of tuberculosis infection
(ARTI). As a conclusion, most of the cases $(83.5 \%)$ were vaccinated with BCG and the PPD test diameter increased significantly in the vaccinated group comparing to the unvaccinated group. It was also observed that as the PPD test diameters became higher, the number of BCG scars increased and there was no difference in sex dependent PPD test reactivity As another result of this study, annual variation in the ratio of ARTI is necessary to be determined by nationwide assessment of PPD test to determine the risk of tuberculosis. ${ }^{6}$

Another study was performed to investigate the relationship between age and previous BCG vaccination with PPD test reactivity to recognize the interpretation of PPD test results in the adult population of Turkey, where the prevalence of tuberculosis is high and a routine BCG vaccination program is performed. ${ }^{11}$ It was also aimed to assess the effect of age and BCG vaccine status on booster reaction. PPD test was used to two healthy adult groups, concluded medical students and elderly people in a retirement home. The students were not significantly different from elderly people concerning positive PPD test response. There was no influence of BCG scars on PPD test reactivity in either group. "The booster effect was observed more commonly in the elderly, but the presence of a BCG scar did not influence the booster effect in either group". This evidence leads to the conclusion that a positive PPD test response and booster reaction in adults in high-prevalence countries may be caused by latent tuberculosis rather than previous vaccination. ${ }^{11}$ A cohort study was performed on some normal-birthweight and low-birthweight infants in Guinea-Bissau. ${ }^{14}$ It was aimed to test the hypothesis that having a scar and a positive PPD test response after vaccination with BCG is related with reduced infant mortality. Children were recruited in randomised trials and BCG scars and PPD test responses were evaluated at 2 and 6 months of age. The evidence which was gained supported previous observations that having a scar and a PPD test response after BCG vaccination is linked with lower mortality risk. It is necessary to consider the possibility of revaccinating scar-negative children. ${ }^{14}$ On the other hand, the Tuberculin skin test cannot differentiate tuberculosis infection from BCG vaccination, and the detection of latent tuberculosis infection does not an approved typical approach. A study in China showed that the new whole-blood interferon $\gamma$ release assay may be a better marker of latent tuberculosis infection than the Tuberculin skin test. ${ }^{17}$

A study in Iran also showed that the recombinant early secretory antigenic target 6 is more specific marker to detect of Mycobacterium tuberculosis infection than Tuberculin skin test. ${ }^{18}$ In our study 38 cases $(32 \%)$ did not have a BCG scar and PPD test was negative in these cases. PPD test indurations diameters were lesser than 3 millimeters in 57 cases $(48 \%)$ and BCG scars diameters were lesser than 2 millimeters in 67 cases (56\%). There was a significant positive relationship between tuberculin test indurations and BCG scar diameter. Intradermal injection is standard technique for the PPD test. ${ }^{19}$ Nevertheless, this method using a traditional needle is frequently hard to accomplish truly and modify testing outcomes. Recently a study showed that the new micro needle MicronJet $600^{\mathrm{TM}}$ applied for the PPD skin test was safe, efficient and lesser painful in healthful adults. A recent study showed that plasma tissue inhibitor of metalloproteinase's 1 might be a new biomarker for the detection of tuberculosis infection [20]. C-Tb (States Serum Institute, Copenhagen, Denmark) is a new special skin test based on CFP10 and ESAT-6 antigens. Ruhwald et al., ${ }^{21}$ investigated the diagnostic potential and safety of $\mathrm{C}-\mathrm{Tb}$ compared with established tests in the contact-tracing setting. They found being unaffected by BCG vaccination status, the $\mathrm{C}-\mathrm{Tb}$ skin test could supply more accurate treatment guidance in settings where the PPD test is widely applied. ${ }^{21}$ Diagnosis of childhood Tuberculosis is 
difficult. Recently a study showed that urinary Lipoarabinomannan (a non-invasive antigen detection test) has good diagnostic value for diagnosis of Tuberculosis in children. Lipoarabinomannan is the chief ingredient of Mycobacterium tuberculosis cell wall. ${ }^{22}$ Singular properties of nanoparticles have permitted their application in diagnosis of tuberculosis using targeting disease biomarkers. Recently in an article El-Samadony et al [23] reviewed state-of-theart knowledge of tuberculosis nanodiagnostics for the last 10 years. They commented tuberculosisnanodiagnostics show promise to be perfect diagnostic tools that can meet the meticulous needs to end the tuberculosis epidemic by $2030 .^{23}$

\section{Conclusion}

Consistent with the results, there is a significant positive relationship between tuberculin test indurations and BCG scar diameter. According to this formula (PPD test indurations diameter $=$ $1.5+1.5 \times$ BCG scar diameter), PPD test indurations is predictable in accordance with BCG scar diameter in 4 year children.

\section{Acknowledgements}

I wish to thank Dr. Sharifi and Ms. Mandana Ghaderi (medical student of Tehran University of Medical Sciences) without whose help this study could not have been completed. We would also to thank all participating children for their cooperation and for their permission to publish this article.

\section{Conflicts of interest}

There are no financial conflicts of interest.

\section{Funding}

None.

\section{References}

1. Pang Y, Kang W, Zhao A, et al. The effect of bacille CalmetteGuérin vaccination at birth on immune response in China. Vaccine. 2015;33(1):209-213.

2. Roth A, Sodemann M, Jensen H, et al. Vaccination technique, PPD reaction and BCG scarring in a cohort of children born in Guinea-Bissau 2000-2002. Vaccine. 2005;23(30):3991-3998.

3. Araujo Z, De Waard JH, De Larrea CF, et al. The effect of Bacille Calmette-Guérin vaccine on tuberculin reactivity in indigenous children from communities with high prevalence of tuberculosis. Vaccine. 2008;26(44):5575-5581.

4. Burl S, Adetifa UJ, Cox M, et al. The tuberculin skin test (TST) is affected by recent $\mathrm{BCG}$ vaccination but not by exposure to non-tuberculosis mycobacteria (NTM) during early life. PLoS One. 2010;5(8):e12287.

5. Hemmati M, Ghadiri K, Rezaei M. Tuberculin Reactivity in School Age Children;Five-year Follow-up in Iran. Iran J Pediatr. 2011;21(1):3944.

6. Yildirim C, Küçük A, Ongüt G, et al. Evaluation of tuberculin reactivity in different age groups with and without BCG vaccination. Mikrobiyol Bul. 2009;43(1):27-35.

7. Mostaan S, Yazdanpanah B, Moukhah R, et al. Adverse effects of BCG vaccine 1173 P2 in Iran:A meta-analysis. Adv Biomed Res. 2016;5:99.
8. Meshkat Z, Teimourpour A, Rashidian S, et al. Immunogenicity of a DNA Vaccine Encoding Ag85a-Tb10.4 Antigens from Mycobacterium Tuberculosis. Iran J Immunol. 2016;13(4):289-295.

9. Fallah R, Naserzadeh N, Ferdosian F, Binesh F. Comparison of effect of kangaroo mother care, breastfeeding and swaddling on Bacillus Calmette-Guerin vaccination pain score in healthy term neonates by a clinical trial. J Matern Fetal Neonatal Med. 2017;30(10):1147-1150.

10. Tatar D, Senol G, Alptekin S, et al. Assessment of Extrapulmonary Tuberculosis in Two Provinces of Turkey. Iran J Public Health. 2016;45(3):305-313.

11. Hizel K, Maral I, Karakus R, et al. The influence of BCG immunisation on tuberculin reactivity and booster effect in adults in a country with a high prevalence of tuberculosis. Clin Microbiol Infect. 2004;10(11):980 983

12. Doosti-Irani A, Ayubi E, Mostafavi E. Tuberculin and QuantiFERONTB-Gold tests for latent tuberculosis:a meta-analysis. Occup Med (Lond). 2016;66(6):437-445.

13. Ayubi E, Doosti-Irani A, Mostafavi E. Do the tuberculin skin test and the QuantiFERON-TB Gold in-tube test agree in detecting latent tuberculosis among high-risk contacts? A systematic review and metaanalysis. Epidemiol Health. 2015;37:e2015043.

14. Timmermann CA, Biering-Sørensen S, Aaby P, et al. Tuberculin reaction and BCG scar:association with infant mortality. Trop Med Int Healt. 2015;20(12):1733-1744.

15. Ten Dam HG. Research on BCG vaccination. Adv Tuberc Res. 1984;21:79-106.

16. Bouros D, Zeros G, Panaretos C, et al. Palpation vs pen method for the measurement of skin tuberculin reaction (Mantoux test). Chest 1991;99(2):416-419.

17. Wu X, Hou Y, Liang Y, et al. Evaluation of a tuberculosis whole-blood interferon $-\gamma$ chemiluminescent immunoassay among Chinese military recruits. Mol Diagn Ther. 2011;15(6):341-346.

18. Moradi J, Mosavari N, Ebrahimi M, et al. Evaluation of Mycobacterium tuberculosis Early Secreted Antigenic Target 6 Recombinant Protein as a Diagnostic Marker in Skin Test. Osong Public Health Res Perspect. 2015;6(1):34-38.

19. Lee HJ, Choi HJ, Kim DR, et al. Safety and efficacy of tuberculin skin testing with microneedle MicronJet600 $0^{\mathrm{TM}}$ in healthy adults. Int J Tuberc Lung Dis. 2016;20(4):500-504.

20. Chen Y, Wang J, Ge P, et al. Tissue inhibitor of metalloproteinases 1, a novel biomarker of tuberculosis. Mol Med Rep. 2017;15(1):483-487.

21. Ruhwald M, Aggerbeck H, Gallardo RV, et al. Safety and efficacy of the $\mathrm{C}-\mathrm{Tb}$ skin test to diagnose Mycobacterium tuberculosis infection, compared with an interferon $\gamma$ release assay and the tuberculin skin test:a phase 3, double-blind, randomised, controlled trial. Lancet Respir Med. 2017;5(4):259-268.

22. Iskandar A, Nursiloningrum E, Arthamin MZ, et al. The Diagnostic Value of Urine Lipoarabinomannan (LAM) Antigen in Childhood Tuberculosis. J Clin Diagn Res. 2017;11(3):EC32-EC35.

23. El-Samadony H, Althani A, Tageldin MA, et al. Nanodiagnostics for tuberculosis detection. Expert Rev Mol Diagn. 2017;17(5):427-443. 